\title{
Respiratory syncytial virus infection accelerates lung fibrosis through the unfolded protein response in a bleomycin-induced pulmonary fibrosis animal model
}

\author{
LINA WANG $^{1}$, WEI CHENG ${ }^{1}$ and ZHIMIN ZHANG ${ }^{2}$ \\ ${ }^{1}$ Department of Respiratory Diseases, Affiliated Hospital of Qingdao University, Qingdao, Shandong 266003, P.R. China; \\ ${ }^{2}$ Department of Respiratory and Intensive Medicine, University of Virginia, Charlottesville, VA 22908, USA
}

Received January 29, 2016; Accepted February 16, 2017

DOI: $10.3892 / \mathrm{mmr} .2017 .6558$

\begin{abstract}
Emerging evidence has demonstrated that endoplasmic reticulum stress (ER) is involved in the pathogenesis of idiopathic pulmonary fibrosis, however, the underlying mechanism remains unclear. Viral infection often triggers a hyperinflammatory response by an expansion of the ER. The present study was designed to observe the role of respiratory syncytial virus infection (RSV)-induced ER stress on lung fibrosis. In order to determine the role of ER stress on the onset and progression of pulmonary fibrosis, mice received an intratracheal combined injection of RSV and bleomycin on day 0 . At day 7, 14 and 21 following combined injection, RSV in the lung tissues was assayed by immunohistochemistry, cellular classification was assayed by direct microscopic observation after Wright staining and the secretion of cytokines in the broncho-alveolar lavage fluid (BALF) was assayed by ELISA. The expression of collagen type I was assayed by immunofluorescence and western blot analysis. The expression of ER stress related proteins was analyzed by western blot. In addition, the correlations of ER-stress related proteins with collagen type-1 were examined. RSV administration resulted in increased inflammation, as demonstrated by increased levels of leukocytes and pro-inflammatory cytokines in the BALF, and increased collagen type-1 deposition in the lung tissues of bleomycin-induced pulmonary fibrosis animal model at 7, 14 and 21 days. RSV promoted the expression of phosphorylated protein kinase R-like endoplasmic reticulum kinase (p-PERK), $78 \mathrm{kDa}$ glucose-regulated protein (GRP78) and activating transcription factor $6 \alpha$ (ATF6 $\alpha$ ), which accelerated the severity and process of fibrosis in bleomycin-induced animal models. The present study provides evidence that
\end{abstract}

Correspondence to: Dr Lina Wang, Department of Respiratory Diseases, Affiliated Hospital of Qingdao University, 16 Jiangsu Road, Qingdao, Shandong 266003, P.R. China

E-mail: drwanglina@outlook.com

key words: endoplasmic reticulum stress, respiratory syncytial virus infection, pulmonary fibrosis
RSV infection accelerated the unfolded protein response and bleomycin-induced lung fibrosis, which may improve our understanding of the pathogenesis of pulmonary fibrosis.

\section{Introduction}

Endoplasmic reticulum (ER) stress is caused by the accumulation of unfolded or misfolded proteins in the ER. Many age-associated diseases have been closely related with ER stress including Alzheimer's and idiopathic pulmonary fibrosis (IPF) $(1,2)$. Furthermore, a series of previous research indicated that increased ER stress may be a key component in the pathogenesis of various age-associated diseases $(3,4)$. In normal conditions, proteins involved in ER stress bind with glucose regulated protein-78 (GRP78), also called immunoglobulin heavy-chain-binding protein. However, under stress due to various factors, the unfolded protein response (UPR) is activated, which contains three important forms of signaling: protein kinase R-like endoplasmic reticulum kinase (PERK) signaling, activating transcription factor $6 \alpha$ (ATF6 $\alpha$ ) signaling and inositol-requiring enzyme 1 (IRE-1) signaling. With accumulation of abnormally folded or improperly assembled proteins in the ER, GRP78 dissociates with the above three proteins, initiating signaling cascades to protect the cell from ER stress. Among the three proteins, PERK senses accumulation of misfolded proteins in the ER and, once activated, undergoes autophosphorylation and dimerization and inactivates the $\alpha$-subunit of eukaryotic translational initiation factor 2 (eIF2a) to attenuate translation of proteins. C/EBP homologous protein (CHOP) is an important molecule involved in PERK signaling cascades and initiates apoptosis (5). Another protein, ATF6 $\alpha$ during ER stress is cleaved by protease and releases its cytoplasmic domain into the cytosol, which binds to cis-acting ER stress response elements and activates the transcription of ER chaperones involved in protein-folding, such as GRP78. The third protein, IRE-1, is a transmembrane protein that has intrinsic serine/threonine kinase and endonuclease activity, which is activated in response to ER stress and cleaves a 26-nucleotide intron sequence from the transcription factor, X-box binding protein (XBP)-1. Spliced XBP-1 translocates to the nucleus and promotes the transcription of target genes, which are related with degradation of misfolded proteins $(5,6)$. 
Emerging evidence has indicated that the ER gets involved in the pathogenesis of idiopathic pulmonary fibrosis, while the involvements of three signaling pathways and the process of lung fibrosis remains unclear. Pulmonary viral infection often triggers a hyperinflammatory response by an expansion of ER stress (7). In the present study, the authors observed the UPR induced by respiratory syncytial virus infection (RSV) infection in the pathogenesis of progressive lung fibrosis.

\section{Materials and methods}

Ethics statement. This study was approved by the Animal Care and Use Committee of Qingdao University (Qingdao, China) and conducted in compliance with the guidelines of the National Institutes of Health for the care and the use of laboratory animals.

Preparation of $R S V$. The strain A2 of human RSV was propagated in HeLa cells (both from the Virology Institute of Wuhan, Wuhan, China) in minimal essential medium supplemented with $2 \%$ heat-inactivated fetal bovine serum (both from Invitrogen; Thermo Fisher Scientific, Waltham, MA, USA). When $75 \%$ of the cells formed syncytia, the cells were harvested and disrupted by sonication using the same culture medium. The suspension was clarified by centrifugation at $10,000 \times \mathrm{g}$ for $15 \mathrm{~min}$ at $4^{\circ} \mathrm{C}$ and stored in aliquots at $-70^{\circ} \mathrm{C}$. Sham inoculum was prepared from uninfected HeLa cell lysate using the same procedure. The viral titer in the inoculums was determined by plaque assay (8) on the same day as the mice were inoculated.

Establishment of RSV+bleomycin-induced animal models. Ten-week-old male C57BL/6 mice ( $\mathrm{n}=80$; weight, 20-25 g) were purchased from the Affiliated Hospital of Qingdao University (Qingdao, China). Mice were given free access to food and water and were maintained with a 12-h light/dark cycle. Mice in the RSV group were endotracheally injected $100 \mu \mathrm{l}$ of PBS and $100 \mu \mathrm{l}$ of RSV $\left(2 \times 10^{6} \mathrm{pfu} / \mathrm{ml}\right)$. Mice in the bleomycin group were endotracheally injected with $100 \mu \mathrm{l}$ bleomycin $(1 \mathrm{mg} / \mathrm{ml})$ in sterile PBS and $100 \mu \mathrm{l} \mathrm{Sham} \mathrm{inoc-}$ ulum. Mice in the RSV+bleomycin group were endotracheally injected RSV $\left(100 \mu \mathrm{l}, 2 \times 10^{6} \mathrm{pfu} / \mathrm{ml}\right)$ and bleomycin $(100 \mu \mathrm{l}$, $1 \mathrm{mg} / \mathrm{ml}$ ) (9). Combined sham inoculums in place of RSV, and PBS in place of bleomycin were used as a control. At 7, 14 or 21 days following injection, the animals were sacrificed, and analyzed. All experimental animals used in the current study were maintained under the protocol approved by the Institutional Animal Care and Use Committee of Qingdao University.

Cellular classification of bronchoalveolar lavage fluid $(B A L F)$. BALFs were centrifuged at $1,000 \times \mathrm{g}$ for $5 \mathrm{~min}$ at room temperature and the cell pellets were smeared onto glass slides. Cells were air dried and stained with Wright stain, which allows differential counting of various cells. In each sample, $\geq 300$ cells per sample were counted under a light microscope.

Cytokines in BALF. Interleukin (IL) $-1,-5$ and -8 productions in BALF were measured using ELISA kits for mouse IL-1 $\beta$
(70-EK201B2/2), mouse IL-5 (70-EK2052) (both from Multi Sciences (Lianke) Biotech Co., Ltd., Hangzhou, China) and mouse IL-8 (kt21138; MoShaKe Biotech Co., Ltd., Wuhan, China) according to the manufacturer's protocol. Briefly, a total of $100 \mu \mathrm{l}$ BALF supernatants were added into a 96-well plate and incubated for $1 \mathrm{~h}$, followed by $100 \mu \mathrm{l}$ enzyme-linked antibodies for $30 \mathrm{~min}$ at $37^{\circ} \mathrm{C}$. Following three washes with washing buffer, the chromogenic reagent was added and incubated for $30 \mathrm{~min}$. $2 \mathrm{M} \mathrm{H}_{2} \mathrm{SO}_{4}$ was added to terminate reaction. Absorbance was determined at $450 \mathrm{~nm}$ using a microplate reader [Tecan Sunrise; Tecan (Shanghai) Trading Co., Ltd., Shanghai, China]. Each sample was repeated three times.

Immunohistochemistry. Mice were sacrificed with an intraperitoneal injection of sodium pentobarbitone $(100 \mathrm{mg} / \mathrm{kg})$ and the right lung lobes were obtained. Lobes were inflated (10\% paraformaldehyde perfusion via trachea), harvested, fixed in $4 \%$ paraformaldehyde overnight, embedded in paraffin and cut into $5 \mu \mathrm{m}$ sections. Endogenous peroxidase was inhibited by soaking tissue sections in $3 \% \mathrm{H}_{2} \mathrm{O}_{2}$. Following rinsing in PBS, sections were incubated with goat serum (Wuhan Boster Biological Technology, Ltd., Wuhan, China) to block nonspecific binding of antibodies, and were then incubated overnight at $4{ }^{\circ} \mathrm{C}$ with rabbit anti-human RSV polyclonal antibodies (orb101035; Biorbyt LLC, San Francisco, CA, USA; dilution, 1:250). Following washing in PBS, the sections were incubated with biotinylated goat anti rabbit IgG (Vector BA-1,000; Vector Laboratories, Inc., Burlingame, CA, USA) at $37^{\circ} \mathrm{C}$ for $1 \mathrm{~h}$, and washed again. Following washing again in PBS, the signal was detected with 3,3'-diaminobenzidine. Omission of primary or secondary antisera was included as a method of providing a control for each biopsy.

Immunofluorescence. Following soaking the sections in $3 \% \mathrm{H}_{2} \mathrm{O}_{2}$ for $15 \mathrm{~min}$ and $10 \mu \mathrm{l}$ of goat serum for $30 \mathrm{~min}$, sections were incubated overnight at $4^{\circ} \mathrm{C}$ with rabbit anti collagen type I polyclonal antibodies (1:150; SAB4500362; Sigma-Aldrich; Merck KGaA, Darmstadt, Germany). Following washing in PBS, the sections were incubated with fluorescein isothiocyanate-labeled goat anti rabbit IgG (1:1,000; A22120; Beyotime Institute of Biotechnology, Haimen, China) for $1 \mathrm{~h}$, and washed again. The slides were finally mounted with mounting media (Thermo Fisher Scientific, Inc.) and examined by fluorescence microscopy.

Western blot analysis. Lung tissues were washed by ice-cold PBS twice and extracted in radioimmunoprecipitation assay buffer (Beijing Dingguo Changsheng Biotechnology Co., Ltd., Beijing, China) containing $1 \mathrm{mM}$ phenylmethylsulfonyl fluoride, $5 \mathrm{mM} \beta$-glycerophosphate and $1 \%$ standard protease inhibitor cocktail (Sigma-Aldrich; Merck KGaA) on ice for $30 \mathrm{~min}$. Protein concentrations were measured by Bradford assay (Thermo Fisher Scientific, Inc.) and adjusted to a final concentration of $10 \mathrm{mg} / \mathrm{ml}$. Lung homogenates $(50 \mu \mathrm{g})$ were subjected to separation by $12 \%$ SDS-PAGE, then transferred to polyvinylidene difluoride membranes (Beijing Dingguo Changsheng Biotechnology Co., Ltd.) and blocked with 5\% skimmed milk. After heat-induced epitope retrieval (Microwave on high for $15 \mathrm{~min}$ ), the slides were 

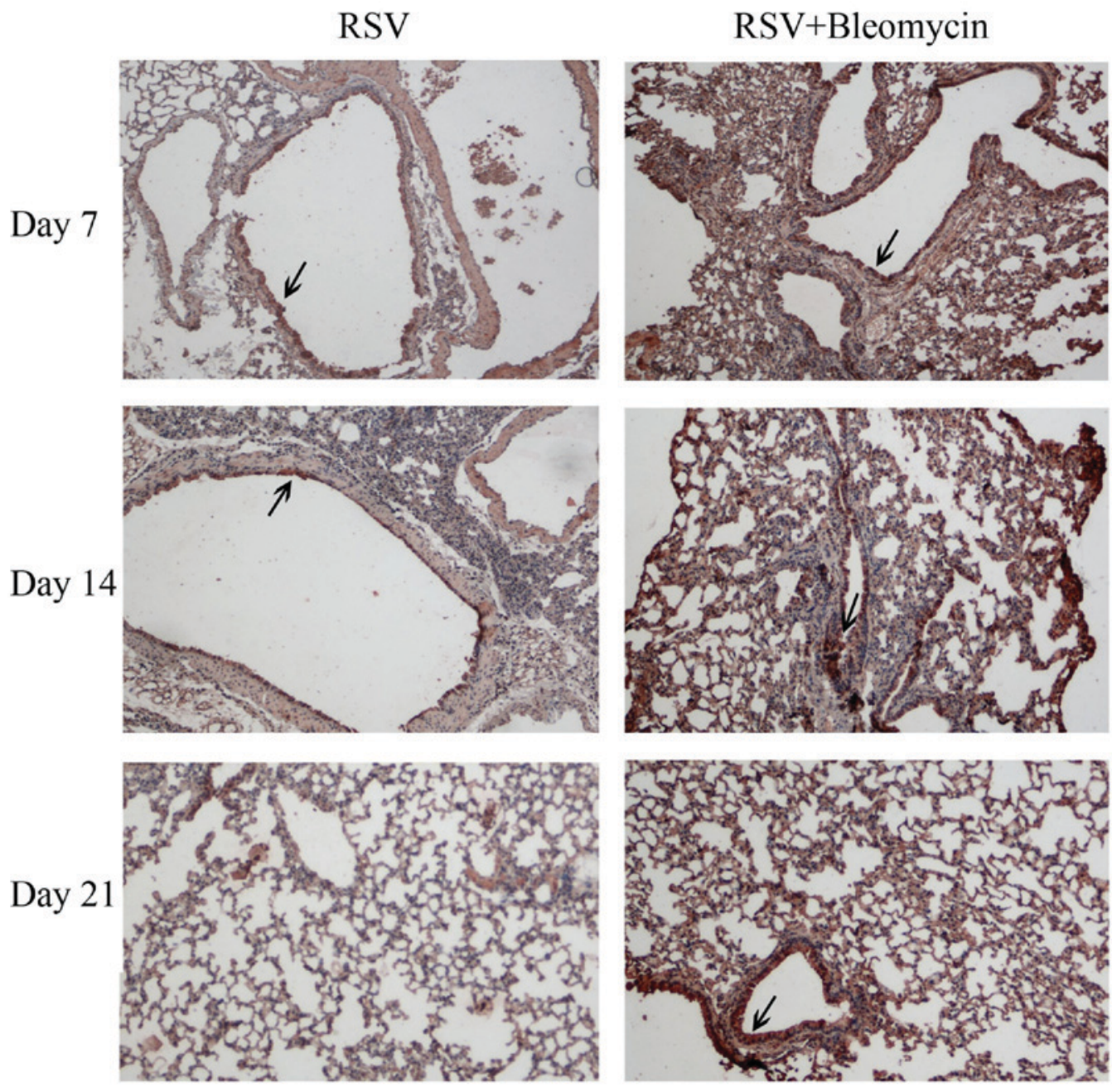

Figure 1. RSV distributed in lung tissues was assayed by immnohistochemistry. RSV mainly distributed in the epithelial cells and peaked at day 7, and then decreased with infection time. In the RSV+bleomycin group, RSV was localized predominantly to alveoli and remained in the lower lungs from day 7 to day 21 (magnification, x100). Arrows showed the positive immunostaining. RSV, respiratory syncytial virus infection.

incubated with rabbit anti-mouse p-PERK (1:150; sc-13073), anti-GRP78 (1:250; sc-13968), anti-CHOP (1:200; sc-575), anti-ATF6 $\alpha$ (1:100; sc-22799), anti-XBP-1 (1:150; sc-7160) (all from Santa Cruz Biotechnology, Inc., Dallas, TX, USA) and rabbit anti-collagen type I polyclonal antibodies (1:200; SAB4500362; Sigma-Aldrich; Merck KGaA) overnight at $4^{\circ} \mathrm{C}$. Blots were developed using corresponding horseradish peroxidase-conjugated goat anti-rabbit antibodies $(1: 2,000$; A0208; Beyotime Institute of Biotechnology) at $37^{\circ} \mathrm{C}$ for $1 \mathrm{~h}$, and detected using an enhanced chemiluminescence system (Amersham ECL Plus; GE Healthcare Life Sciences, Chalfont, UK). Band intensities were quantified by Image Gauge (version 3.4) software with the LAS-1,000 plus system (Fujifilm Holdings Corporation, Tokyo, Japan). $\beta$-actin served as a loading control.

Statistical analysis. The statistical significance of the data was analyzed using the SPSS software (version 14.0; SPSS Inc., Chicago, IL, USA). Data were expressed as means \pm standard deviation. Statistical analyses were performed using an unpaired Student's t-test for the comparison of data from different treatment groups. $\mathrm{P}<0.05$ was considered to indicate a statistically significant difference.

\section{Results}

$R S V$ persistence in bleomycin-induced pulmonary fibrosis animal models. The results of immnohistochemistry indicated that RSV (dark brown staining) was evident in the airway epithelium and peaked at day 7 and decreased with infection time in the RSV alone group. In RSV+bleomycin group, the RSV infection was localized predominantly to alveoli and was persistent in the lungs from day 7 to day 21 (Fig. 1). No viruses were observed in uninfected animals (data not shown).

$R S V$ promoted inflammation of bleomycin-induced pulmonary fibrosis animal models. The lungs of mice infected with RSV presented significant increases in the numbers of eosinophils at day $7(\mathrm{P}<0.01)$, when compared with the control group, before returning to normal levels. In addition, the bleomycin group demonstrated obvious increases in macrophages and neutrophils at day 7 (both $\mathrm{P}<0.01$ ), only macrophages at day $14(\mathrm{P}<0.01)$, when compared with the control group before returning to normal levels of all red blood cell types at day 21. However, the RSV+bleomycin group exhibited obvious increases in macrophages, neutrophils and lymphocytes at day 7, 14 and $21(\mathrm{P}<0.01)$ when compared with 
A
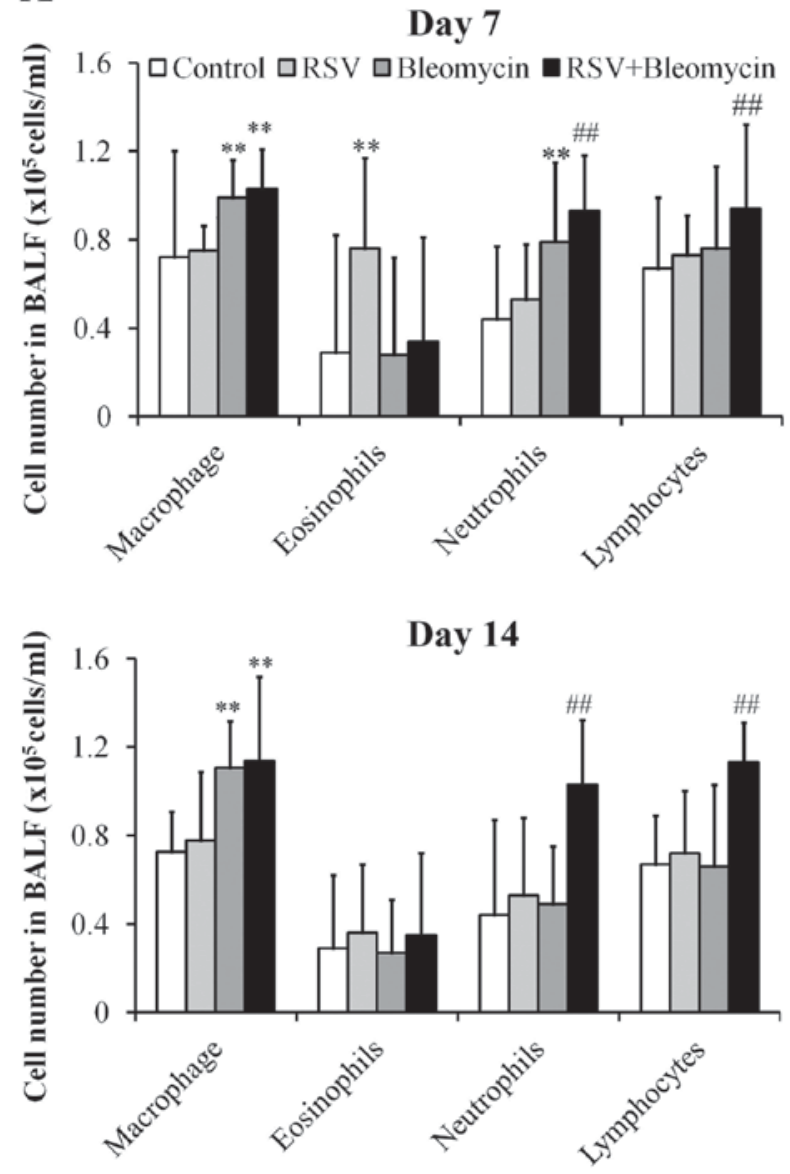

Day 21

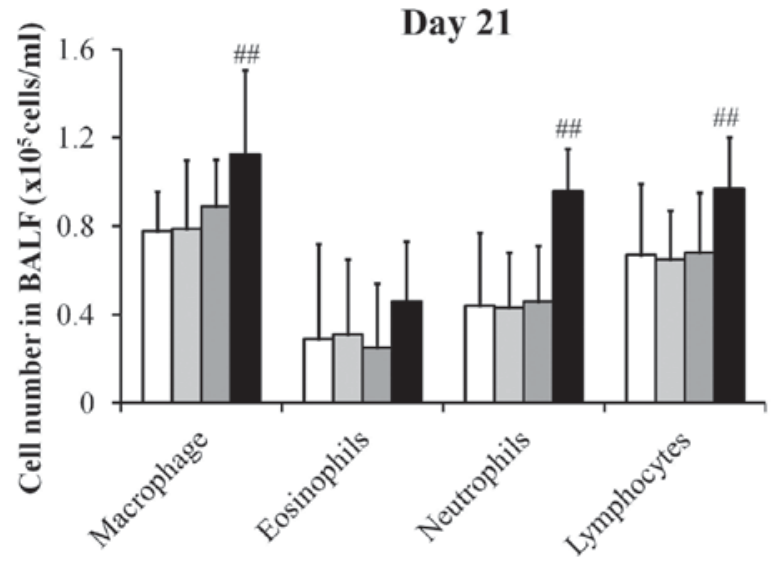

B

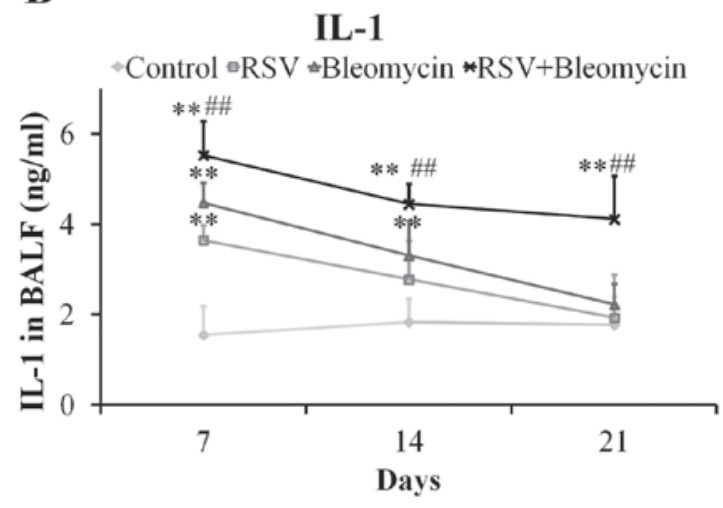

IL-5

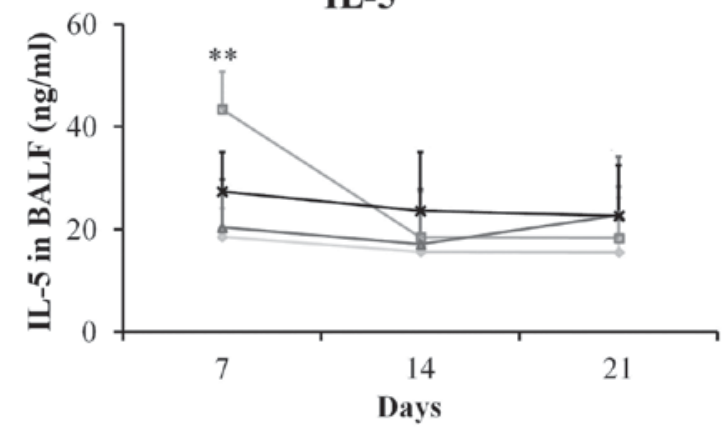

IL-8

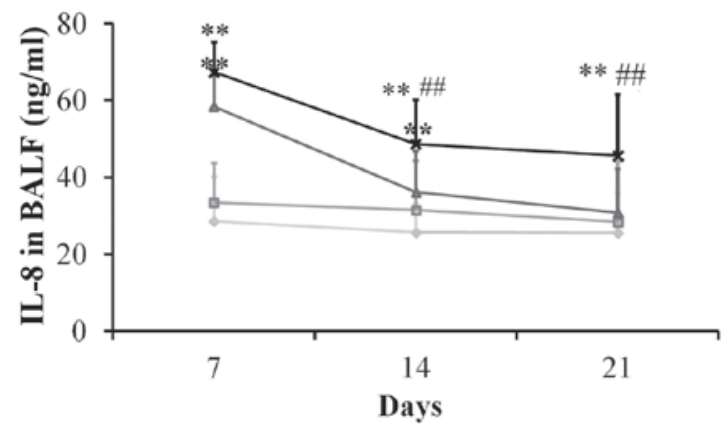

Figure 2. Cellular classification and cytokine secretions were assayed by direct microscopic observation following (A) Wright staining and (B) ELISA. The RSV group demonstrated significant increases in the numbers of eosinophils at day 7. The bleomycin group presented significant increases in macrophages and neutrophils at day 7, and in macrophages only at day 14. However, the RSV+bleomycin group presented significant increases in macrophages, neutrophils and lymphocytes from day 7 to day 21 . RSV promoted the production of IL-1 and -5 at day 7 . Bleomycin induced release of IL-1 and -8 at day 7 and day 14 . However, the RSV+bleomycin group demonstrated significant increases in IL-1 and -8 from day 7 to day 21 , when compared with the control group ( $\mathrm{n}=6$, ${ }^{* *} \mathrm{P}<0.01$ vs. control; ${ }^{\# \#} \mathrm{P}<0.01$ vs. bleomycin). RSV, respiratory syncytial virus infection; IL, interleukin.

bleomycin group (Fig. 2A). In pursuit of the effects of RSV on the process of bleomycin-induced pulmonary fibrosis, the authors investigated the production of the pro-inflammatory cytokines IL-1, -5 and -8 in BALF. As demonstrated in Fig. 2B, RSV promoted the production of IL-1 and -5 at day 7 (both $\mathrm{P}<0.01$ ), when compared with the control group, before returning to normal levels. Bleomycin induced release of IL-1 and -8 at day 7 and day 14 (all $\mathrm{P}<0.01$ ), when compared with the control group, before returning to normal levels at day 21. However, RSV+bleomycin group presented obvious increases in IL-1 and -8 at days 7, 14 and 21 (all $\mathrm{P}<0.01$ ), when compared with control or the bleomycin group.

$R S V$ promoted collagen synthesis of bleomycin-induced pulmonary fibrosis animal models. Excessive production and deposition of extracellular matrix components 
A
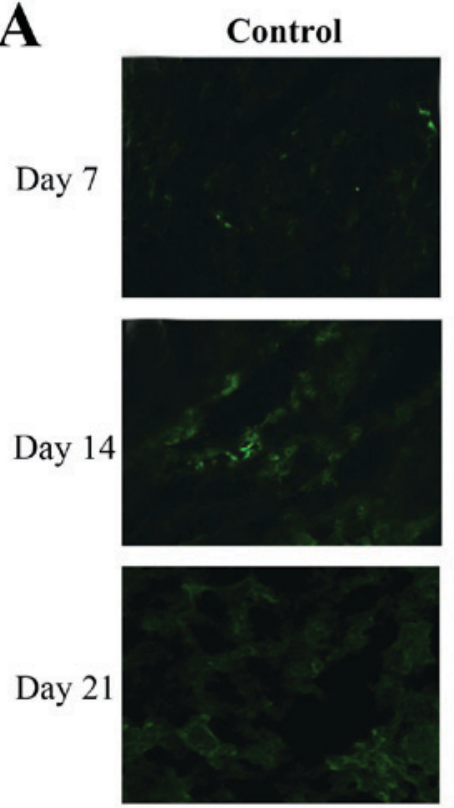

B

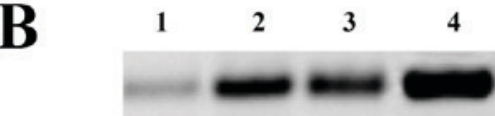

Day 7

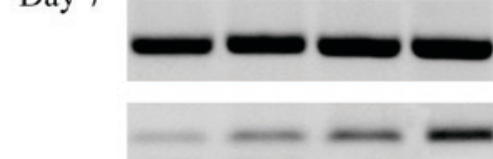

Day 14
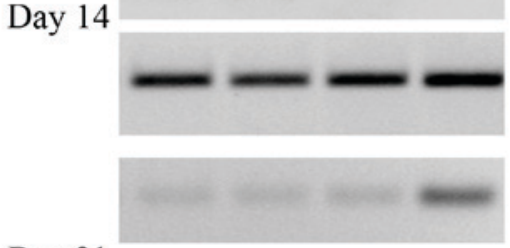

Day 21

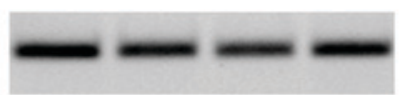

Collagen type-1

RSV
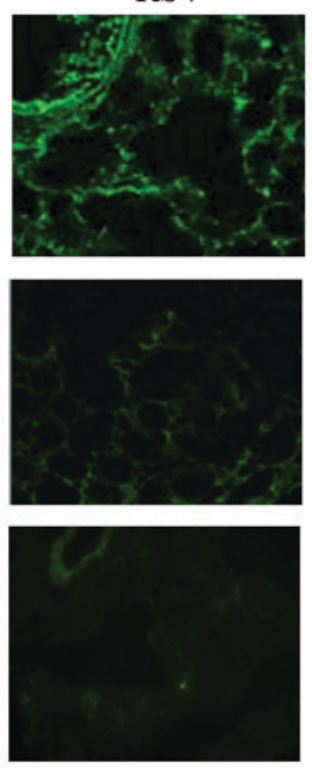

Collagen type-1

Actin

Collagen type-1

Actin

Actin
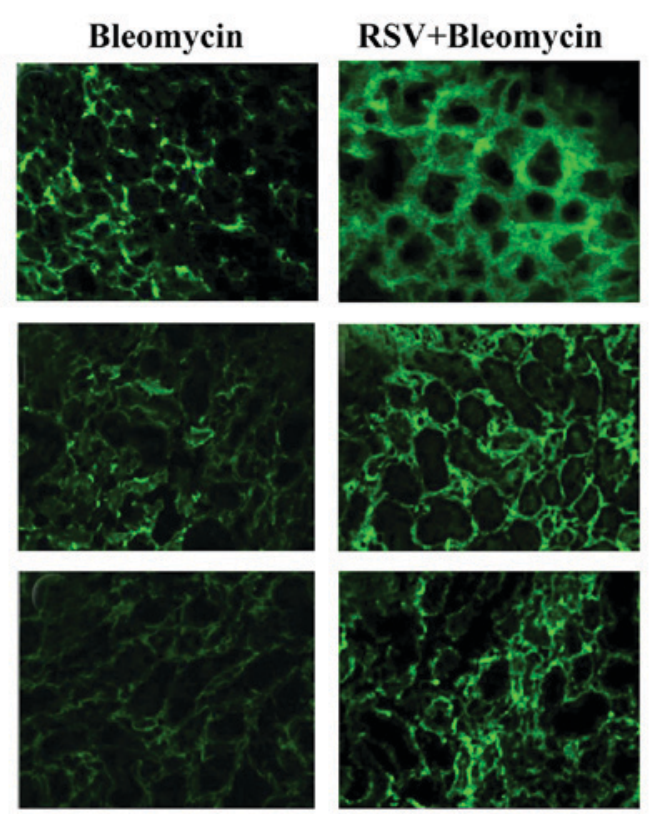

C

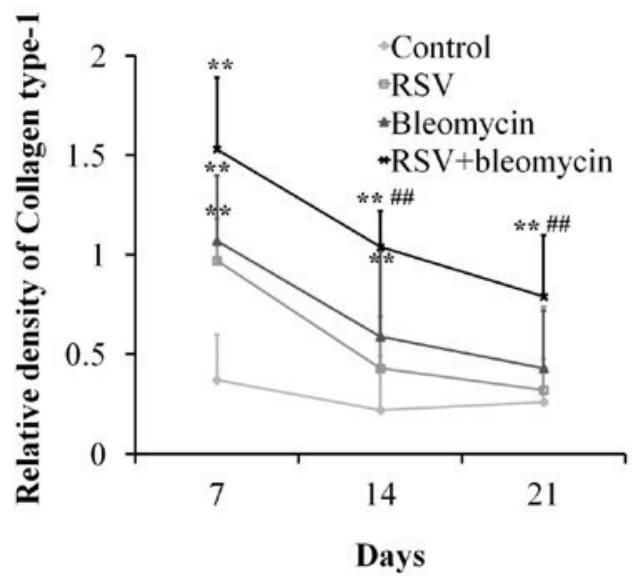

Figure 3. Collagen type-1 in lung tissues was assayed by (A) immunofluorescence (magnification, x100) and (B and C) western blot analyses. RSV or bleomycin alone promoted the accumulation of collagen type I in the lungs from day 7, before decreasing in a time dependent manner. However, RSV promoted the accumulation of collagen type I at different time points in bleomycin-induced pulmonary fibrosis animal models. $\beta$-actin was used as a loading control. ( $\mathrm{n}=6$, ${ }^{* *} \mathrm{P}<0.01$ vs. control; ${ }^{\#} \mathrm{P}<0.01$ vs. bleomycin). RSV, respiratory syncytial virus infection.

is crucial for the development of pulmonary fibrosis. As demonstrated in Fig. 3, immunofluorescence (Fig. 3A) and western blot analyses (Fig. 3B and C) identified that RSV or bleomycin alone promoted the accumulation of collagen type I in the lungs at day 7, when compared with the control group (Fig. 3C; $\mathrm{P}<0.01$ ); these effects decreased with time. However, RSV+bleomycin promoted more collagen type I over the time course compared with bleomycin alone.

RSV promoted expression of p-PERK, ATF6 $\alpha$ and GRP78 in bleomycin-induced pulmonary fibrosis animal models. Using western blot analysis, GRP78, p-PERK, CHOP, XBP-1 and ATF6 $\alpha$ were detectable at low levels in control lung tissues at days 7, 14 and 21 (Fig. 4). Mice treated with RSV demonstrated significantly elevated levels of p-PERK, ATF6 $\alpha$ and GRP78 at day 7 compared with those of controls and returned to normal levels at days 14 and 21 (Fig. 4; all $\mathrm{P}<0.01)$. Mice treated with bleomycin demonstrated significantly elevated levels of CHOP, XBP-1, GRP78 and ATF6 $\alpha$ at day 7, CHOP, XBP-1 and GRP-78 at day 14, when compared with those of controls, before returning to normal levels at day 21 (Fig. 4; all $\mathrm{P}<0.01$ ). Mice treated with combined RSV+bleomycin demonstrated significantly elevated levels of p-PERK, XBP-1, ATF6 $\alpha$ and GRP78 from day 7 to day 21, when compared to the control group (Fig. 4; all $\mathrm{P}<0.01)$.

In order to further identify the effects of ER stress-related proteins induced by RSV in the lung fibrosis, the correlations of p-PERK, ATF6 $\alpha$ and GRP78 with accumulation of collagen type-1 were examined. The results indicated that p-PERK, $(\mathrm{P}=0.0093)$, ATF6 $\alpha(\mathrm{P}=0.0097)$ and GRP78 $(\mathrm{P}=0.0089)$ were closely related with accumulation of collagen type-1 (Fig. 5). 

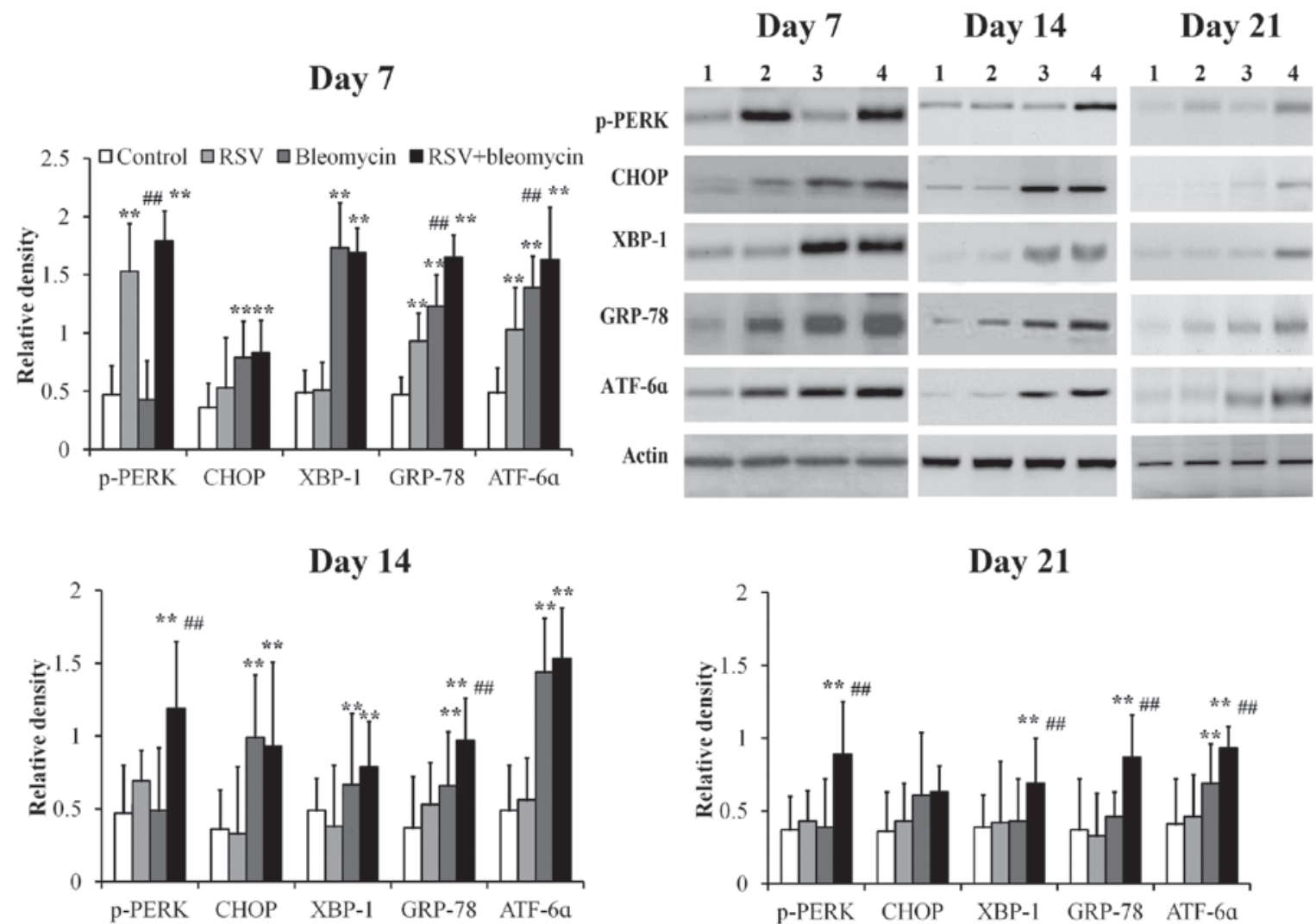

Figure 4. The expressions of ER stress-related proteins in lung tissues were assayed at days 7, 14 and 21 by western blot analysis. The relative density of all tested ER stress related proteins was weak in controls from day 7 to day 21. p-PERK, ATF6 $\alpha$ and GRP78 were upregulated at day 7 in RSV-infected lung tissues, when compared with the control group. CHOP, XBP-1, GRP78 and ATF6 $\alpha$ proteins were upregulated at day 7 and CHOP and GRP-78 were upregulated at day 14 in bleomycin-treated lung tissues, compared to the control group. However, in RSV-infected bleomycin-induced animal models, p-PERK, XBP-1, ATF6 $\alpha$ and GRP78 were upregulated from day 7 to day $21 .{ }^{* *} \mathrm{P}<0.01$ vs. control; ${ }^{* \#} \mathrm{P}<0.01$ vs. bleomycin. ER, endoplasmic reticulum; RSV, respiratory syncytial virus infection, CHOP, C/EBP homologous protein; XBP-1, X-box binding protein; GRP-78, 78 kDa glucose-regulated protein; ATF6 $\alpha$, activating transcription factor $6 \alpha$; PERK, protein kinase R-like endoplasmic reticulum kinase.
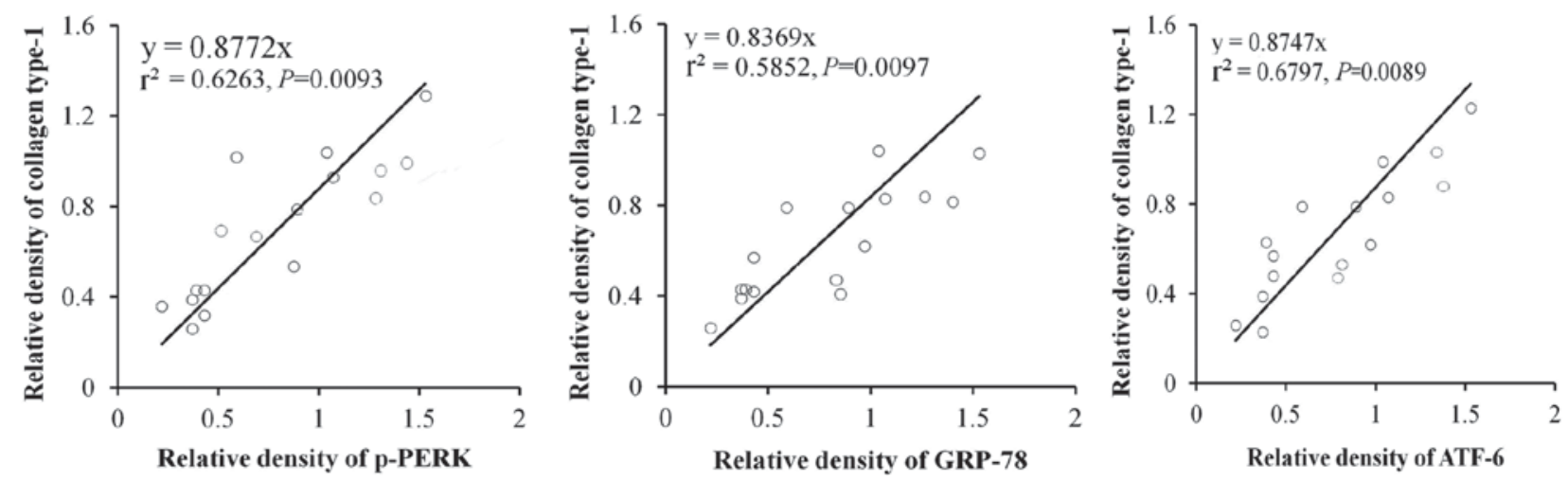

Figure 5. Correlations of endoplasmic reticulum stress proteins with an accumulation of collagen type-1. p-PERK, ATF6 $\alpha$ and GRP78 were closely related with accumulation of collagen type-1. PERK, protein kinase R-like endoplasmic reticulum kinase; ATF6 $\alpha$, activating transcription factor $6 \alpha$; GRP-78, 78 kDa glucose-regulated protein.

\section{Discussion}

The involvement of ER stress in the pathogenesis of pulmonary fibrosis has been researched in previous years $(10,11)$. Lung microbiota is crucially important to maintain the normal function of the lungs, and respiratory infection is a very common factor that may induce ER stress of lung diseases $(12,13)$. To study the effects of viral infection on the onset and progression of lung fibrosis, a bleomycin-induced pulmonary fibrosis animal model was established, as it is a mature model for studying the process of lung fibrosis by observing inflammation and collagen deposition (14). Within the animal models, persistent RSV immunoreactivity was observed until day 21 in the lung tissues, following acute infection (day 7) following the combined administration of RSV and bleomycin. 
Many inflammatory cells, inflammatory media and cytokines are involved in lung inflammation. Among then, IL-5 is considered as the key chemokine for the recruitment and activation of eosinophils and IL-8 for neutrophils (15). In the present study, the authors demonstrated that, when co-administered, bleomycin and RSV promoted the counts of various leukocytes and release of IL-1 and -8, when compared with pulmonary fibrosis induced by bleomycin alone.

The production and deposition of collagen and other extracellular matrix components is a characteristic feature of pulmonary fibrosis (16). Using RSV or bleomycin alone was self-limiting to induce collagen deposition in the lungs. However, beyond promoting inflammation following acute bleomycin administration, RSV was also able to promote tissue fibrosis at earlier time points, lasting for a longer period of time.

The activation of ER stress and UPR were first observed in patients with familial interstitial pneumonia (FIP) with a mutation in surfactant protein C (SFTPC) (17). The presence of UPR was demonstrated in type II alveolar epithelial cells, which specifically express SFTPC. In a subsequent study, it was demonstrated that prominent ER stress in alveolar epithelial cells was also observed in patients with FIP without SFTPC mutation, as well as in patients with sporadic IPF (18). Potential causes of ER stress in the lungs may include exposure to inhaled particles or smoke or infections $(17,19)$. It was indicated in the present study that infection with RSV leads to the development of ER stress in the lungs and the subsequent exaggerated lung fibrosis in bleomycin-induced pulmonary fibrosis, whereas RSV alone only developed mild reversible infections. This suggested that activation of ER stress serves a role in the severity of the fibrotic process. Of the three key transducers of the UPR, the roles of PERK, ATF6 $\alpha$ and IRE1-XBP1 signaling are all involved in exaggerated lung fibrosis (20).

In summary, the current study demonstrated that RSV exaggerated the fibrotic process induced by bleomycin. Further studies investigating the mechanism of RSV administration on the course of fibrotic lung diseases would be of great benefit to the field. Based on these results, the authors suggest that ER stress induced by RSV infection are involved in pulmonary fibrosis.

\section{References}

1. De Felice FG and Lourenco MV: Brain metabolic stress and neuroinflammation at the basis of cognitive impairment in Alzheimer'sdisease. Front Aging Neurosci 7: 94, 2015.

2. Pluquet O, Pourtier A and Abbadie C: The unfolded protein response and cellular senescence. A review in the theme: Cellular mechanisms of endoplasmic reticulum stress signaling in health and disease. Am J Physiol Cell Physiol 308: C415-C425, 2015.

3. Reinhardt S, Schuck F, Grösgen S, Riemenschneider M, Hartmann T, Postina R, Grimm M and Endres K: Unfolded protein response signaling by transcription factor XBP-1 regulates ADAM10 and is affected in Alzheimer's disease. FASEB J 28: 978-997, 2014.

4. Tanjore H, Blackwell TS and Lawson WE: Emerging evidence for endoplasmic reticulum stress in the pathogenesis of idiopathic pulmonary fibrosis. Am J Physiol Lung Cell Mol Physiol 302: L721-L729, 2012.
5. Bettigole SE and Glimcher LH: Endoplasmic reticulum stress in immunity. Annu Rev Immunol 33: 107-138, 2015.

6. Mera K, Kawahara K, Tada K, Kawai K, Hashiguchi T, Maruyama I and Kanekura T: ER signaling is activated to protect human HaCaT keratinocytes from ER stress induced by environmental doses of UVB. Biochem Biophys Res Commun 397: 350-354, 2010.

7. Reed M, Morris SH, Owczarczyk AB and Lukacs NW: Deficiency of autophagy protein Map1-LC3b mediates IL-17-dependent lung pathology during respiratory viral infection via ER stress-associated IL-1. Mucosal Immunol 8: 1118-1130, 2015.

8. Cheung MB, Sampayo-Escobar V, Green R, Moore ML, Mohapatra S and Mohapatra SS: Respiratory syncytial virus-infected mesenchymal stem cells regulate immunity via interferon beta and indoleamine-2,3-dioxygenase. PLoS One 11: e0163709, 2016.

9. Tan Y, Yang T, Liu S, Liu H, Xiang Y, Qu F, Li H and Qin X: Infection with respiratory syncytial virus alters peptidergic innervation in the lower airways of guinea-pigs. Exp Physiol 93: 1284-1291, 2008

10. Mo XT, Zhou WC, Cui WH, Li DL, Li LC, Xu L, Zhao P and Gao J: Inositol-requiring protein 1 -X-box-binding protein 1 pathway promotes epithelial-mesenchymal transition via mediating snail expression in pulmonary fibrosis. Int J Biochem Cell Biol 65: 230-238, 2015.

11. Hoffman SM, Tully JE, Nolin JD, Lahue KG, Goldman DH, Daphtary N, Aliyeva M, Irvin CG, Dixon AE, Poynter ME and Anathy V: Endoplasmic reticulum stress mediates house dust mite-induced airway epithelial apoptosis and fibrosis. Respir Res 14: 141, 2013.

12. Wang L, Wu G, Qin X, Ma Q, Zhou Y, Liu S and Tan Y: Expression of nodal on bronchial epithelial cells influenced by lung microbes through DNA methylation modulates the differentiation of T-helper cells. Cell Physiol Biochem 37: 2012-2022, 2015.

13. Tan YR, Peng D, Chen CM and Qin XQ: Nonstructural protein-1 of respiratory syncytial virus regulates HOX gene expression through interacting with histone. Mol Biol Rep 40: 675-679, 2013.

14. Ashley SL, Jegal Y, Moore TA, van Dyk LF, Laouar Y and Moore BB: $\gamma$-Herpes virus-68, but not Pseudomonas aeruginosa or influenza A (H1N1), exacerbates established murine lung fibrosis. Am J Physiol Lung Cell Mol Physiol 307: L219-L230, 2014.

15. Zhang JS, Tan YR, Xiang Y, Luo ZQ and Qin XQ: Regulatory peptides modulate adhesion of polymorphonuclear leukocytes to bronchial epithelial cells through regulation of interleukins, ICAM-1 and NF-kappaB/IkappaB. Acta Biochim Biophys Sin (Shanghai) 38: 119-128, 2006.

16. Tilbury K, Hocker J, Wen BL, Sandbo N, Singh V and Campagnola PJ: Second harmonic generation microscopy analysis of extracellular matrix changes in human idiopathic pulmonary fibrosis. J Biomed Opt 19: 086014, 2014.

17. Lawson WE, Crossno PF, Polosukhin VV, Roldan J, Cheng DS, Lane KB, Blackwell TR, Xu C, Markin C, Ware LB, et al: Endoplasmic reticulum stress in alveolar epithelial cells is prominent in IPF: Association with altered surfactant protein processing and herpesvirus infection. Am J Physiol Lung Cell Mol Physiol 294: L1119-L1126, 2008.

18. Lawson WE, Cheng DS, Degryse AL, Tanjore H, Polosukhin VV, $\mathrm{Xu} \mathrm{XC}$, Newcomb DC, Jones BR, Roldan J, Lane KB, et al: Endoplasmic reticulum stress enhances fibrotic remodeling in the lungs. Proc Natl Acad Sci USA 108: 10562-10567, 2011.

19. Zhao H, Yang J, Shan L and Jorgensen ED: Measuring the impact of cigarette smoke on the UPR. Methods Enzymol 489: 147-164, 2011.

20. Nanua S, Sajjan U, Keshavjee S and Hershenson MB: Absence of typical unfolded protein response in primary cultured cystic fibrosis airway epithelial cells. Biochem Biophys Res Commun 343: 135-143, 2006. 